ارزيابى تحمل به خشكى در زنوتيڤهاى كَنده دوروه با استفاده از

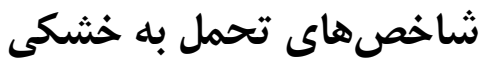

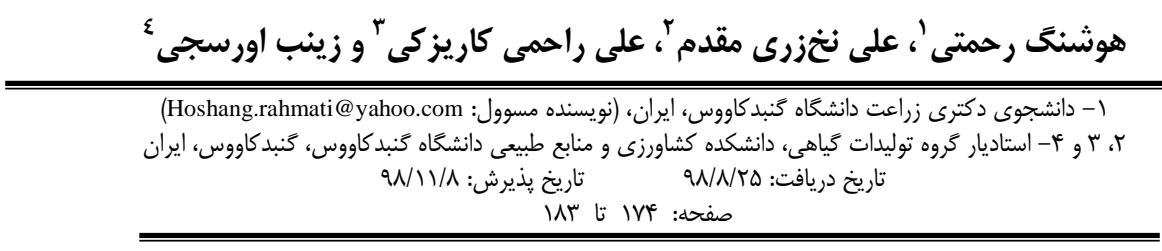

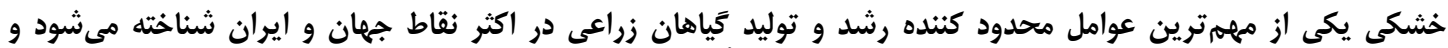

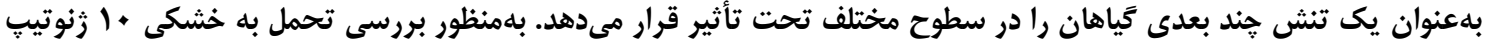

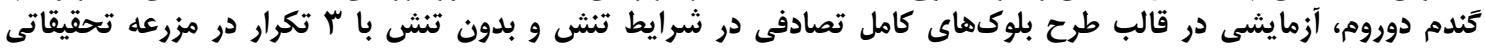

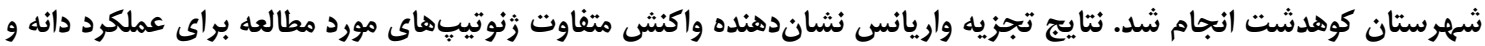

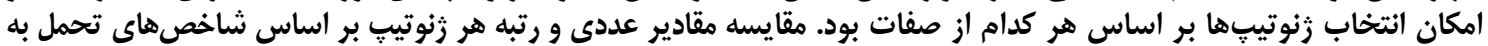

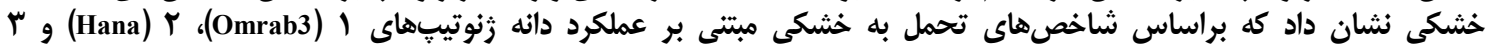
(Aria(stork))

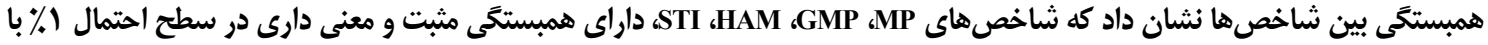

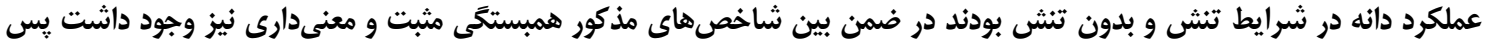

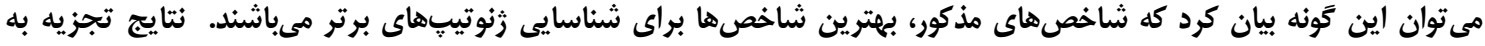

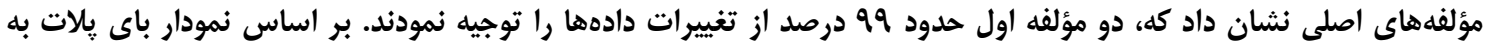

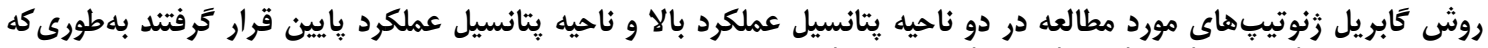

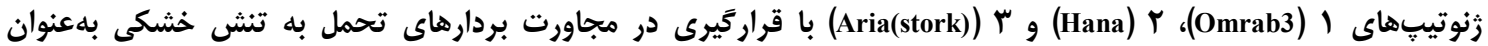

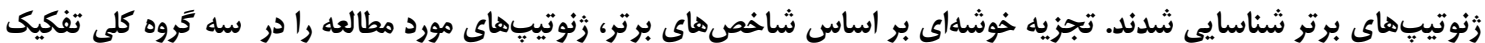

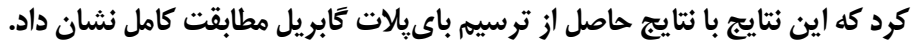

$$
\text { وازههاى كليدى: كَندم ، باى بلات، تنش }
$$

اجتنابنايذير است. محصولات كندم دوروم بلهصورت ماكارونى

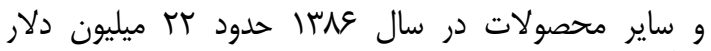

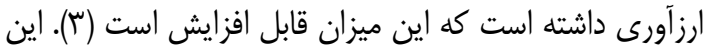

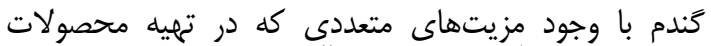

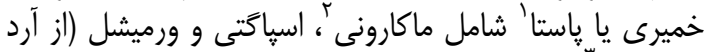

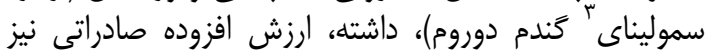

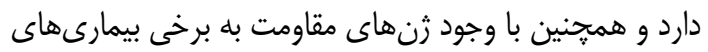

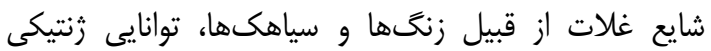

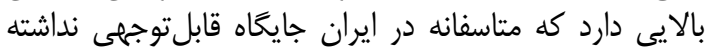

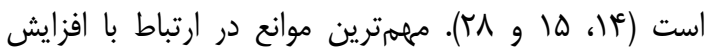

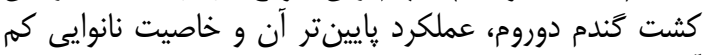

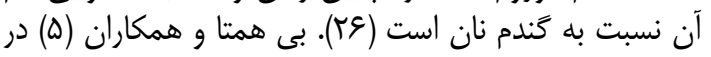

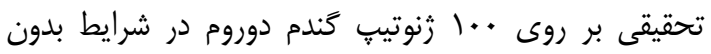

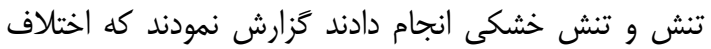

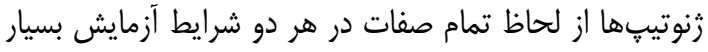

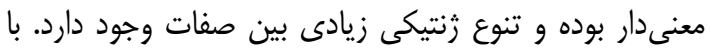

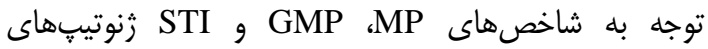

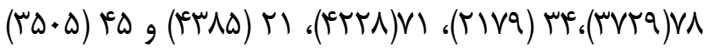

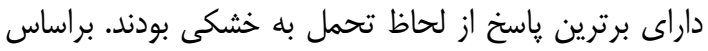

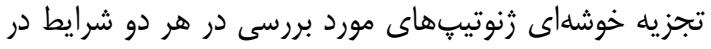
جهار گروه قرار گَرفتند.

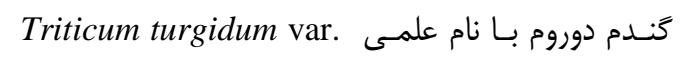

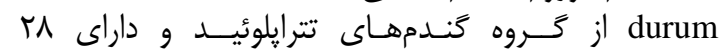

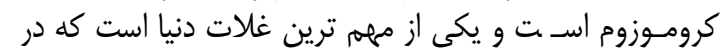

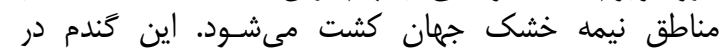

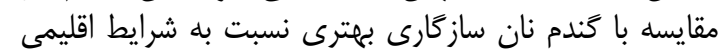

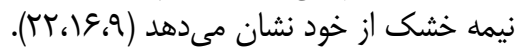

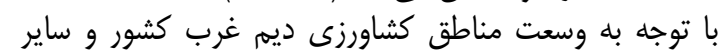

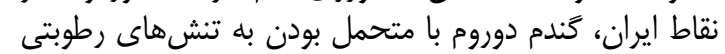

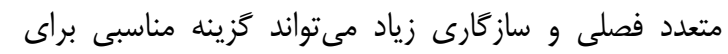

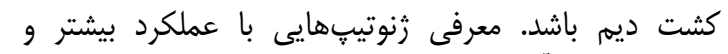

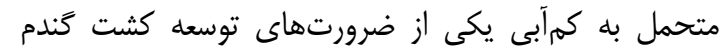

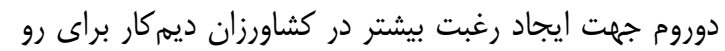

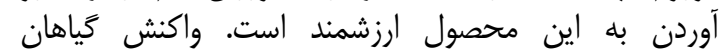

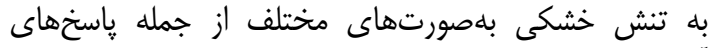

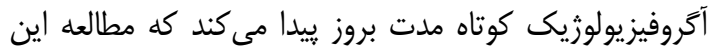

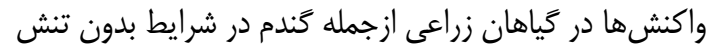

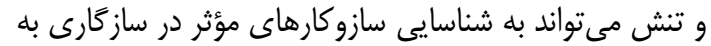
تنش خشكى كمك نمايد (1).

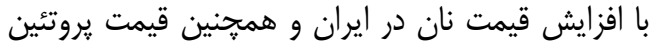

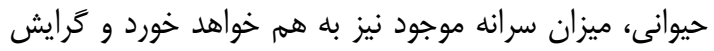
به مصرف جايكزين ارزانتر بان ارزش غذائ بردي بيشتر 


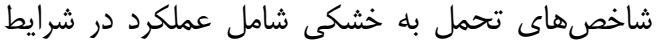

فاقد تنش (YP)، عملكرد در شرايط تنش (YS) (YS)، $\mathrm{SSI}=1-(\bar{Y} s / \bar{Y} p)$

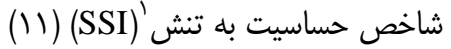

$\mathrm{TOL}=\mathrm{Yp}-\mathrm{Ys}$

$$
\text { شاخص تحمل (TOL) (r) (T) }
$$

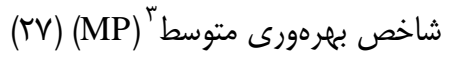

$\mathrm{MP}=(\mathrm{Ys}+\mathrm{Yp}) / 2$

$\mathrm{GMP}=\sqrt{ }(\mathrm{Ys})\left(\mathrm{Y}_{\mathrm{p}}\right)$

ميانكَين هندسى بهرورى (') (GMP)

شاخص ميانگين هارمونيك

$\mathrm{HAM}=2(\mathrm{Yp} \times \mathrm{Ys}) /(\mathrm{Yp}+\mathrm{Ys})$

شاخص تحمل به تنش

$\mathrm{STI}=(\mathrm{Yp}) \times(\mathrm{Ys}) /(\bar{Y} p)^{2}$

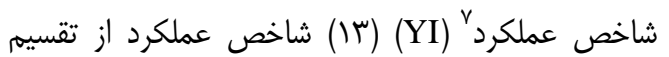

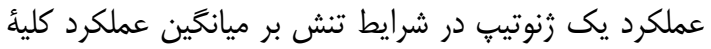

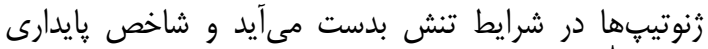

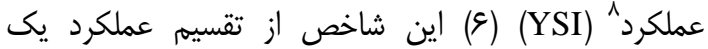

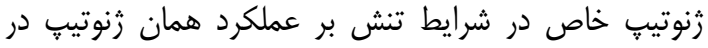
شرايط بدون تنش محاسبه مى خرد درد.

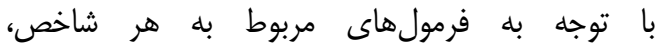

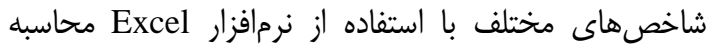

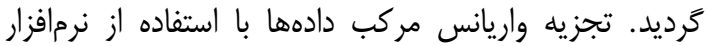
SAS

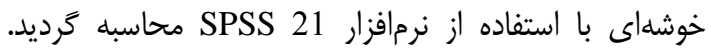

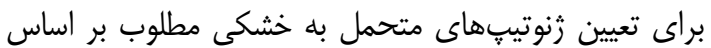

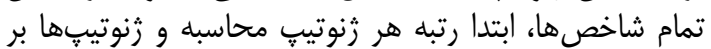

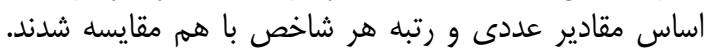

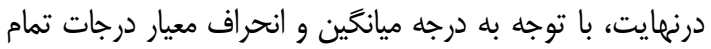

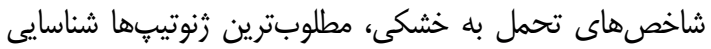

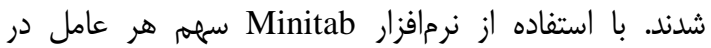

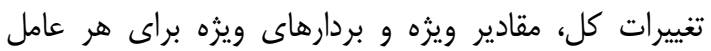

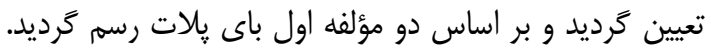

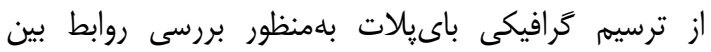

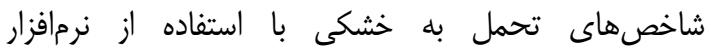
Minitab 16

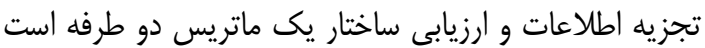

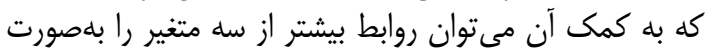
يكجا بررسى كرد (ساب).

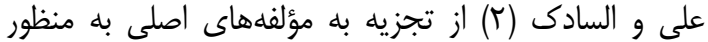

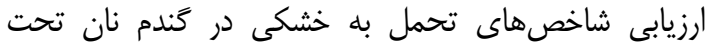

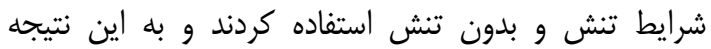

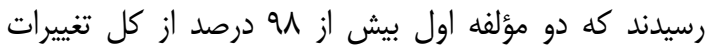

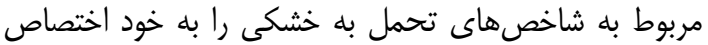
دادهاند. لذا هدف از تحقيق فوق بررسى تحمل خشكى رنوتيبهاى

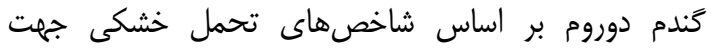

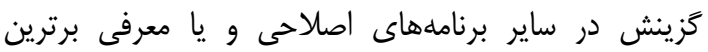

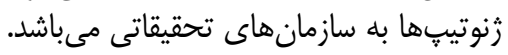

\section{مواد و روشها}

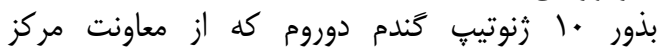

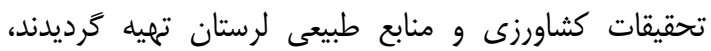

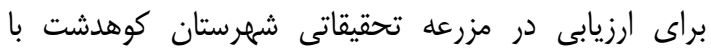

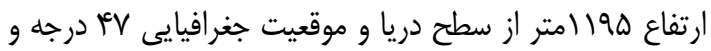

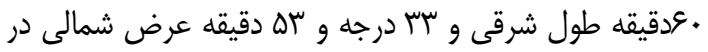

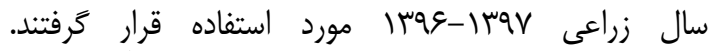

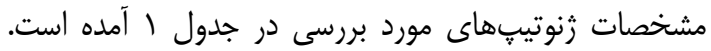

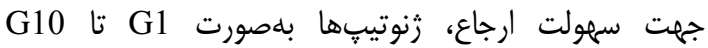

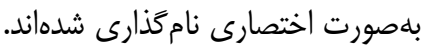

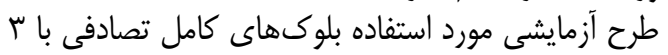

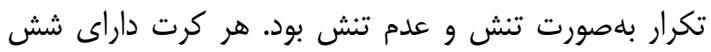

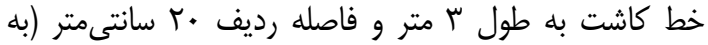

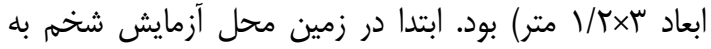

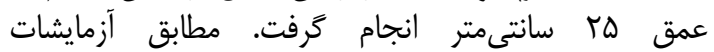

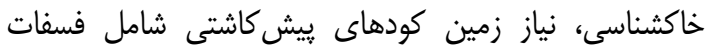

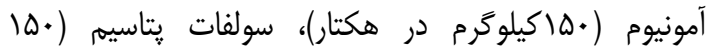

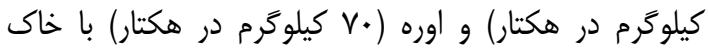

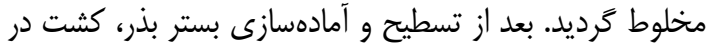

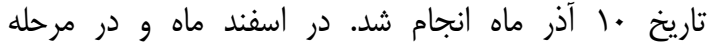

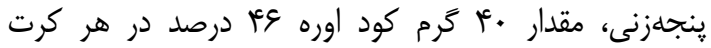

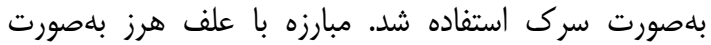

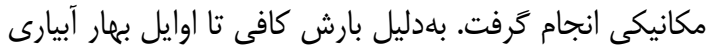

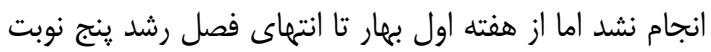

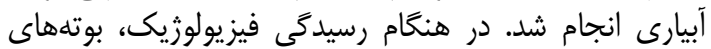

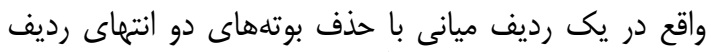

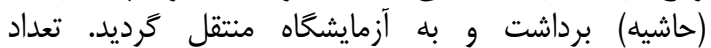

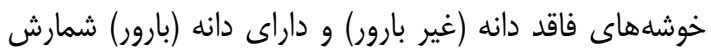

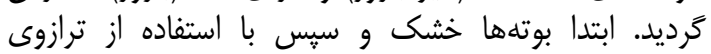

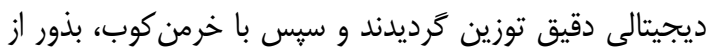
بوتهها جدا و عملكرد دانه اندازهميرى شد.

\begin{tabular}{llll}
\hline 1- Stress Susceptibility Index & 2- Tolerance & 3- Mean productivity & 4- Geometric Mean Productivity \\
5- Harmonic Mean & 6- Stress Tolerance Index & 7- Yield Index & 8- Yield Stability Index
\end{tabular}


Table 1.Characteristics of the tested genotypes

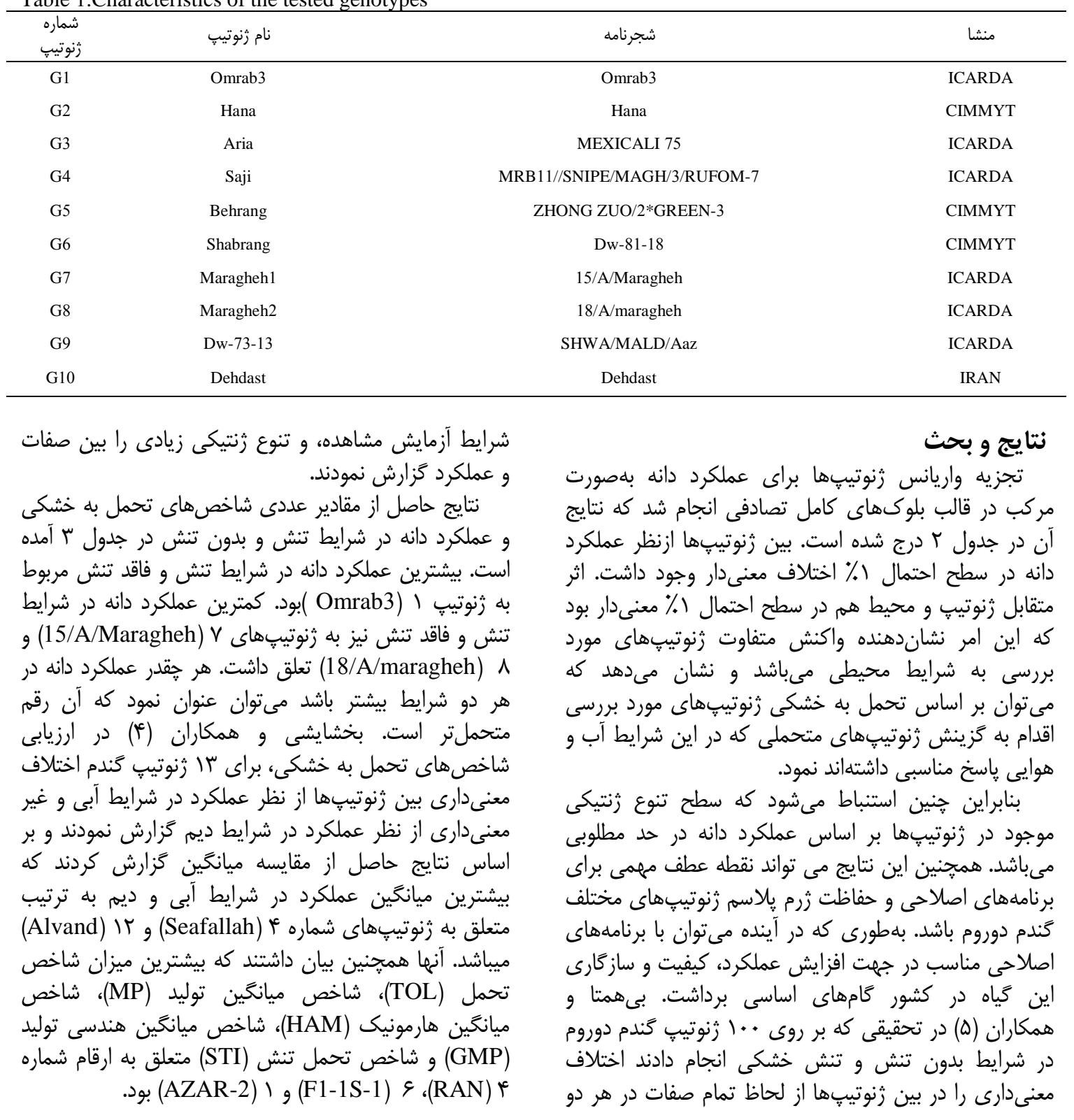

جدول r- ميانگين مربعات عملكرد دانه در زنوتيڤهاى گندم دوروم مورد بررسى Table 2. Mean squares of grain yield in the durum wheat genotypes studied

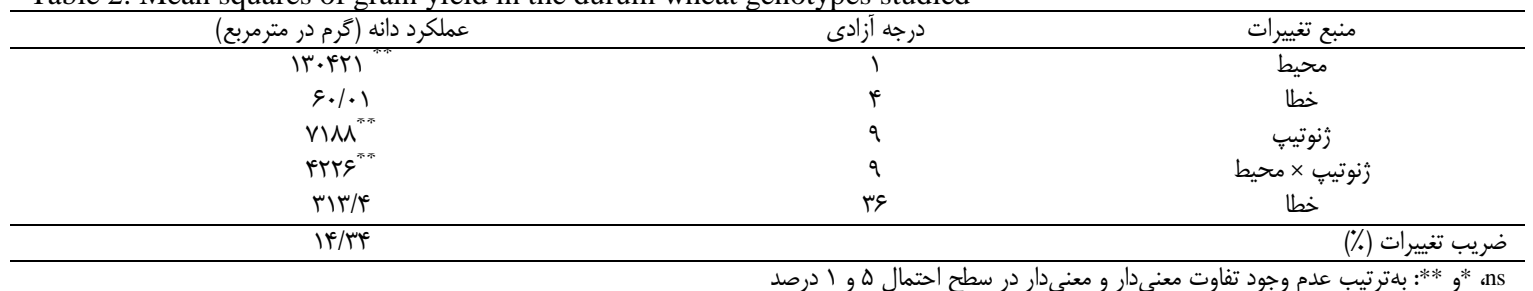


Table 3. Numerical values of drought tolerance indices studied in durum wheat genotypes

\begin{tabular}{|c|c|c|c|c|c|c|c|c|c|c|}
\hline زنوتيب & $\mathrm{Yp}$ & Ys & TOL & MP & GMP & HAM & SSI & STI & YI & YSI \\
\hline 1 & $\mathrm{TV} / \mathrm{V}$ & $1.8 / \pi$ & $|V| / \Delta$ & $199 / \cdot$ & $\mathrm{IV} / \mathrm{A}$ & $1 \Delta \Gamma / V$ & $1 / 48$ & $1 / \cdot r I$ & $1 / r \Delta F$ & אוא/. \\
\hline$r$ & $T r F / T$ & $q . / v \wedge$ & | & $\mid \Delta V / \Delta$ & IFT/V & $1 \pi q / r$ & $1 / \cdot 18$ & $\cdot / V \cdot f$ & I/MAT & $\cdot / \kappa \cdot \Delta$ \\
\hline r & $197 / \%$ & $9 \% / . q$ & $99 / M^{2}$ & IFT/V & גואו & $\mid r \Delta / \Delta$ &.$/ 941$ & .1819 & I/TIT & $\cdot / F A F$ \\
\hline f & $\mid F T /$. & rه/. & $\Delta S / q \Delta$ & $11 \pi / \Delta$ & $1.9 / 9$ & $1.9 / 4$ & . & . Fiv & $1 / 1 \cdot v$ & .1099 \\
\hline$\Delta$ & $r \Pi / r$ & $\Delta S / N S$ & $\mid \Delta E / F$ & $\mid r \varphi /$. & $1.9 / 9$ & $19 / 9$ & זr/ז &.$/ 410$ & $\cdot / V^{c}$ & . rsq \\
\hline 9 & $W E / V$ & $81 / \cdot r^{4}$ & $\mid r \Delta / V$ & $\mid r r / q$ & $1.9 / 1$ & $9 Y / \cdot$ & $1 / T r V$ & . &.$/ V 9 \Delta$ & . TTV \\
\hline$v$ & $\Lambda \Delta / \cdot \Delta$ & G./Ar & TE/TT & $V r / q r$ & VI/qu & V./qu &.$/ 019$ &.$/ 1 V q$ & . /var & $\cdot / V I \Delta$ \\
\hline$\wedge$ & $1 F q / 1$ & $\Delta r / \& q$ & Q & $1.1 / \mathrm{V}$ & $\Lambda Q / \Delta T$ & VN/Ar & $1 / 1 V r$ &.$/ T W V$ & .1999 & $\cdot / T \Delta V$ \\
\hline 9 & $11 \pi / 1$ & $V g / F$ & & $q \uparrow / v V$ & $9 \% / 99$ & $91 / \pi{ }^{4}$ & . $/ 09$. & . &.$/ 999$ &.$/ 9 V G$ \\
\hline 1. & $\| N / F$ & $\Lambda F / r \Delta$ & $r F / / \phi$ & $1.1 / \mu$ & ११/М & $q / \mp \Delta$ & . & . & $1 / .9 v$ &.$/ V I T$ \\
\hline
\end{tabular}

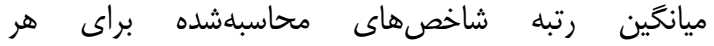

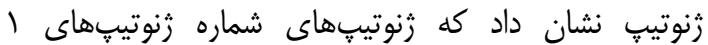
(Hana )rOmrab3)

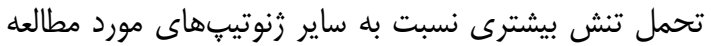
هستند (جدول تأ).

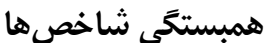

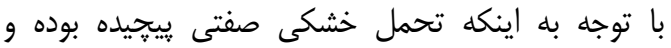

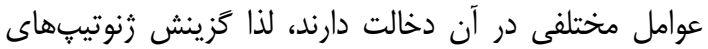

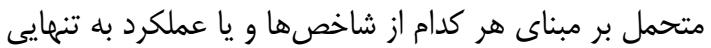

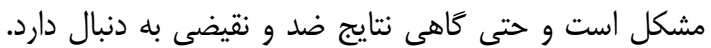

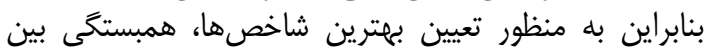

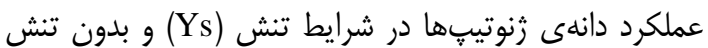

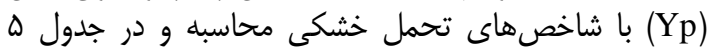
درج شده است. شاخصهايى كه در دو محيط تنش خشكى داسى و فاقد تنش

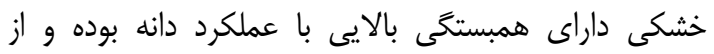

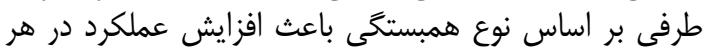

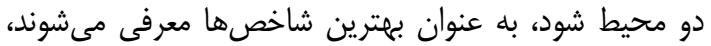

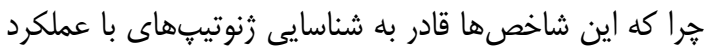

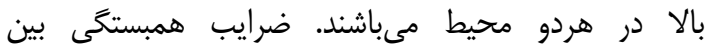

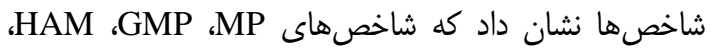
STI

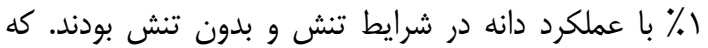

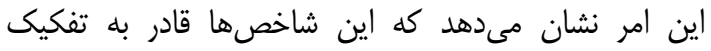

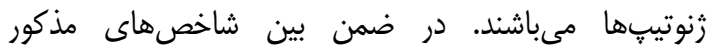

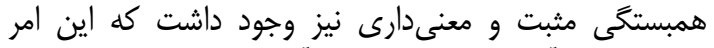

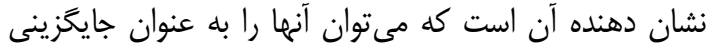

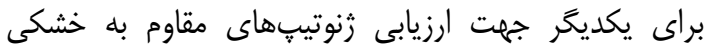

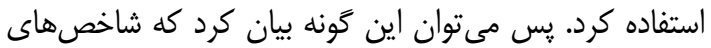

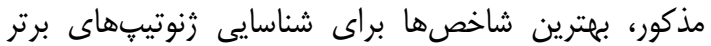
مىباشند. شاخصهاى TOL و SSI با يكديخر همبستخى مثبت و معنىدار نشان دادند. همبستخى شاخص TOL TOL TOا عملكرد دانه

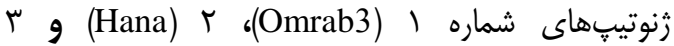
GMP و از نظر شاخص هاى HAM (Aria(stork))

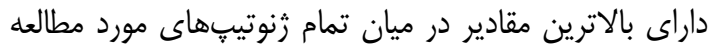

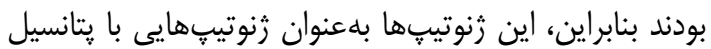

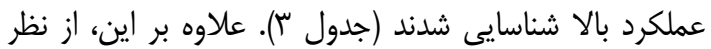

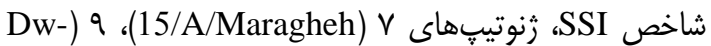

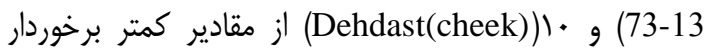

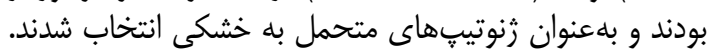

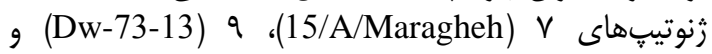

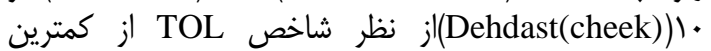

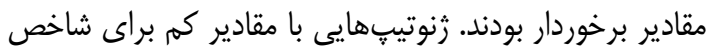

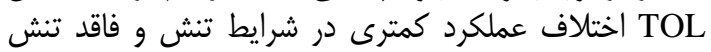

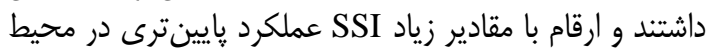

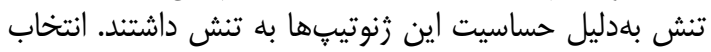

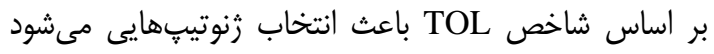

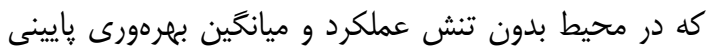

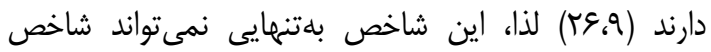

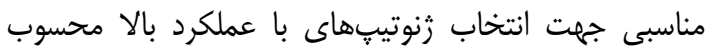

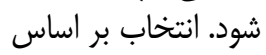
شاخص SSI نيز باعث انتخاب زنوتيبهايى مىشود كه

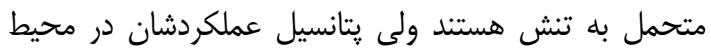

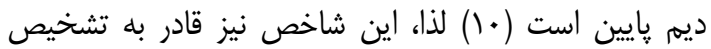

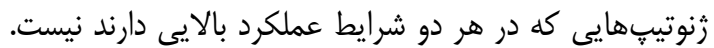

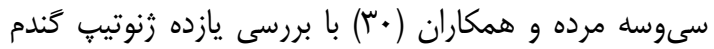

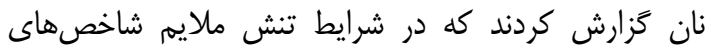

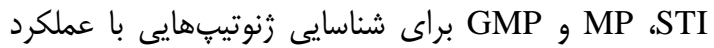
بالا در شرايط تنش و بلدون تنش مناسب هستند رئن

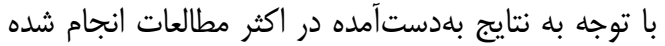

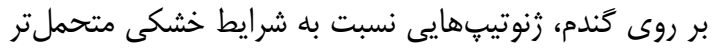

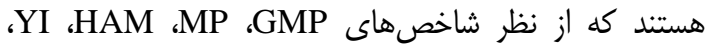
SSI داراى بيشترين مقادير و از نظر شاخصهای STI و

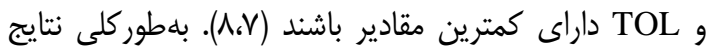

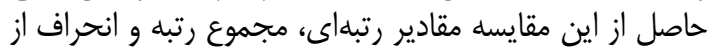




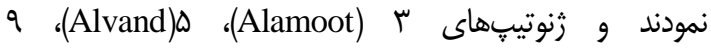

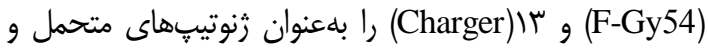
رنوتيبهاى (Gaspard)V)، ("Ald"s)

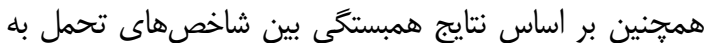

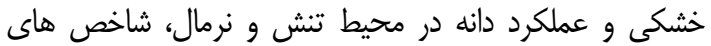

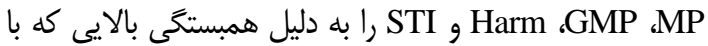

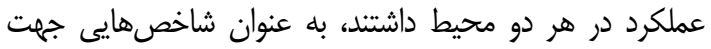
انتخاب زنوتيبهاى داراى يُّانسيل عملكرد بالا در هر دو محيط

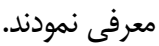

در شرايط تنش مثبت و غيرمعنى دار و با عملكرد در شرايط

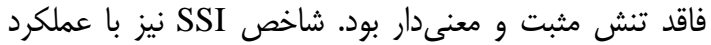

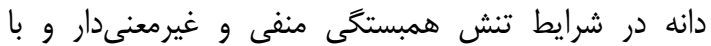

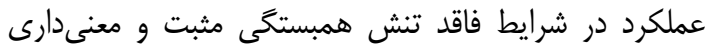

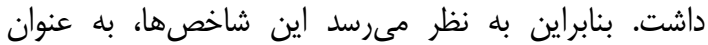

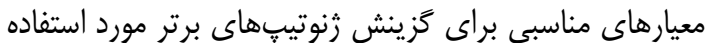

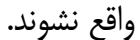

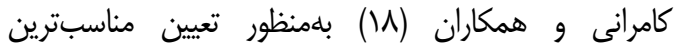

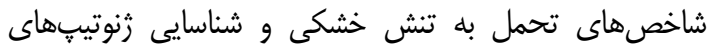

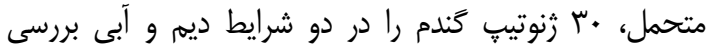

جدول ع - رتبه، ميانگين رتبه و انحراف معيار شاخصهاى تحمل به خشكى مورد مطالعه در زنوتيِهاى كَندم دوروم Table 4. Rank, mean rank and standard deviationof drought tolerance indices studied in durum wheat genotypes

\begin{tabular}{|c|c|c|c|c|c|c|c|c|c|c|c|c|c|}
\hline رنوتيب & Yp & Ys & TOL & MP & GMP & HAM & SSI & STI & YI & YSI & مجموع & ميانخين & انحراف معيار \\
\hline r & $r$ & r & $\wedge$ & $r$ & r & $r$ & 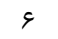 & r & r & 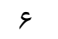 & f. & f & T/KG \\
\hline f & V & f & f & 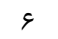 & f & f & $f$ & f & f & f & et & $r / r$ & سצ/. \\
\hline$\Delta$ & r & 9 & 9 & f & $\Delta$ & $\wedge$ & 1. & $\Delta$ & 9 & 1. & vq & $V / q$ & سא/ץ \\
\hline$\checkmark$ & 1. & $\wedge$ & 1 & 1. & 1. & 1. & 1 & 1. & $\wedge$ & 1 & $\varepsilon$. & 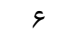 & $r / T r$ \\
\hline$\wedge$ & 9 & 1. & $\Delta$ & v & 9 & 9 & $\wedge$ & 9 & 1. & $\wedge$ & سו & $\Lambda / \mu$ & $1 / 4 q$ \\
\hline 9 & 9 & 8 & r & 9 & $\wedge$ & v & r & $\wedge$ & $\varepsilon$ & r & $\Delta F$ & Q/S & $k / 4 I$ \\
\hline 1. & $\wedge$ & $\Delta$ & $r$ & $\wedge$ & $\checkmark$ & $\Delta$ & $r$ & v & $\Delta$ & r & $F \Delta$ & $c / \Delta$ & عس/ץ \\
\hline
\end{tabular}

Table 5. Correlation of drought tolerance indices

\begin{tabular}{|c|c|c|c|c|c|c|c|c|c|}
\hline & Yp & Ys & TOL & MP & GMP & HAM & SSI & STI & YI \\
\hline Yp & $1 / \cdot$ & & & & & & & & \\
\hline Ys & $\cdot / 4 V$ & $1 / \cdot$. & & & & & & & \\
\hline TOL & $\cdot / 90^{* *}$ &.$/ 11$ & $1 /$. & & & & & & \\
\hline MP & $\cdot / q v^{* *}$ & $\cdot \mid 9 \omega^{* *}$ & $\cdot / N^{* *}$ & $1 /$. & & & & & \\
\hline GMP &.$/ 9 *^{* *}$ & $\cdot / v^{* * *}$ & $\cdot / N^{* * *}$ &.$/ 9 \gamma^{* *}$ & $1 / .$. & & & & \\
\hline HAM & $\cdot / \mathrm{V} \wedge^{* *}$ & $\cdot / 9 *^{* *}$ & $\cdot / \Delta V$ &.$/ 9 *^{* * *}$ & $\cdot / 9 \vee^{* *}$ & $1 / .$. & & & \\
\hline SSI & $\cdot / V^{* *}$ & $-\cdot / 19$ & $. / 9)^{* * *}$ &.$|9|^{* * *}$ & .148 & G/T & $1 / \cdot$ & & \\
\hline STI & $\cdot / \wedge q^{* *}$ & $\cdot / v^{* *}$ & $\cdot / r r^{* *}$ &.$/ 99^{* *}$ &.$/ 99^{* *}$ &.$/ 98^{* * *}$ & TET & $1 / .$. & \\
\hline YI &.$/ 4 V$ & $1^{* * *}$ &.$/ 1 \Lambda$ & $\cdot \mid q \omega^{* *}$ & $\cdot / v^{* *}$ & $. / 9 . * *$ &.$- / 19$ & $\cdot / \mathrm{Vq}^{* * *}$ & $1 /$. \\
\hline YSI & $-\cdot / V V^{* *}$ & .119 & $-. / 9)^{* *}$ &.$-|9|^{* *}$ &.$- / 49$ & g & $-1 / \cdot$. &.$- / 4 T$ &.$/ 19$ \\
\hline
\end{tabular}

اول حدود 99 درصد كل تغييرات دادهها را بيـان نمودنـد لــذا،

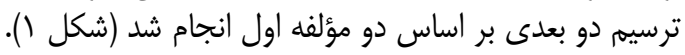

\section{تجزيه به مؤلفههاى اصلى}

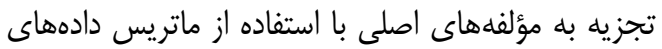

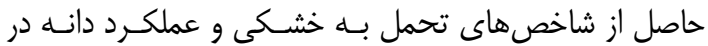

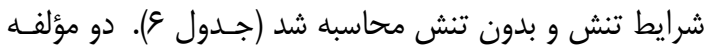


جدول צ- مقادير ويثه، سهم تجمعى و بردارهاى ويزه شاخصهاى تحمل به خشكى و عملكرد دانه در شرايط تنش و فاقد تنش خشكى , خلى Table 6. Eigen values, cumulative contribution and eigenvectors of drought resistance and grain yield indices under

\begin{tabular}{|c|c|c|}
\hline 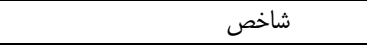 & مؤلفه اول & مؤلفه دوم \\
\hline Yp &.$/ 999$ & $\cdot / r \Delta$ \\
\hline MP &.$/ 9 \vee 9$ & $\cdot / r+1$ \\
\hline TOL &.$/ 941$ & $-\cdot / \mu 1 \Delta$ \\
\hline GMP &.$/ 911$ & 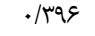 \\
\hline STI & $. / 9 .$. &.$/ 419$ \\
\hline HAM & $\cdot / 1 \cdot 1$ & $\cdot \mid \Delta \wedge \varepsilon$ \\
\hline SSI & $\cdot / V 1$ & س س \\
\hline YSI & $-\cdot / \mathrm{r} /$ & س س \\
\hline YI &.$/ 494$ & . /NEV \\
\hline Ys &.$/ F q 4$ & . /NEV \\
\hline درصد واريانس & $99 / 90$ & $r T / V I$ \\
\hline درصد واريانس تجمعى & $98 / 90$ & ११/४ \\
\hline
\end{tabular}

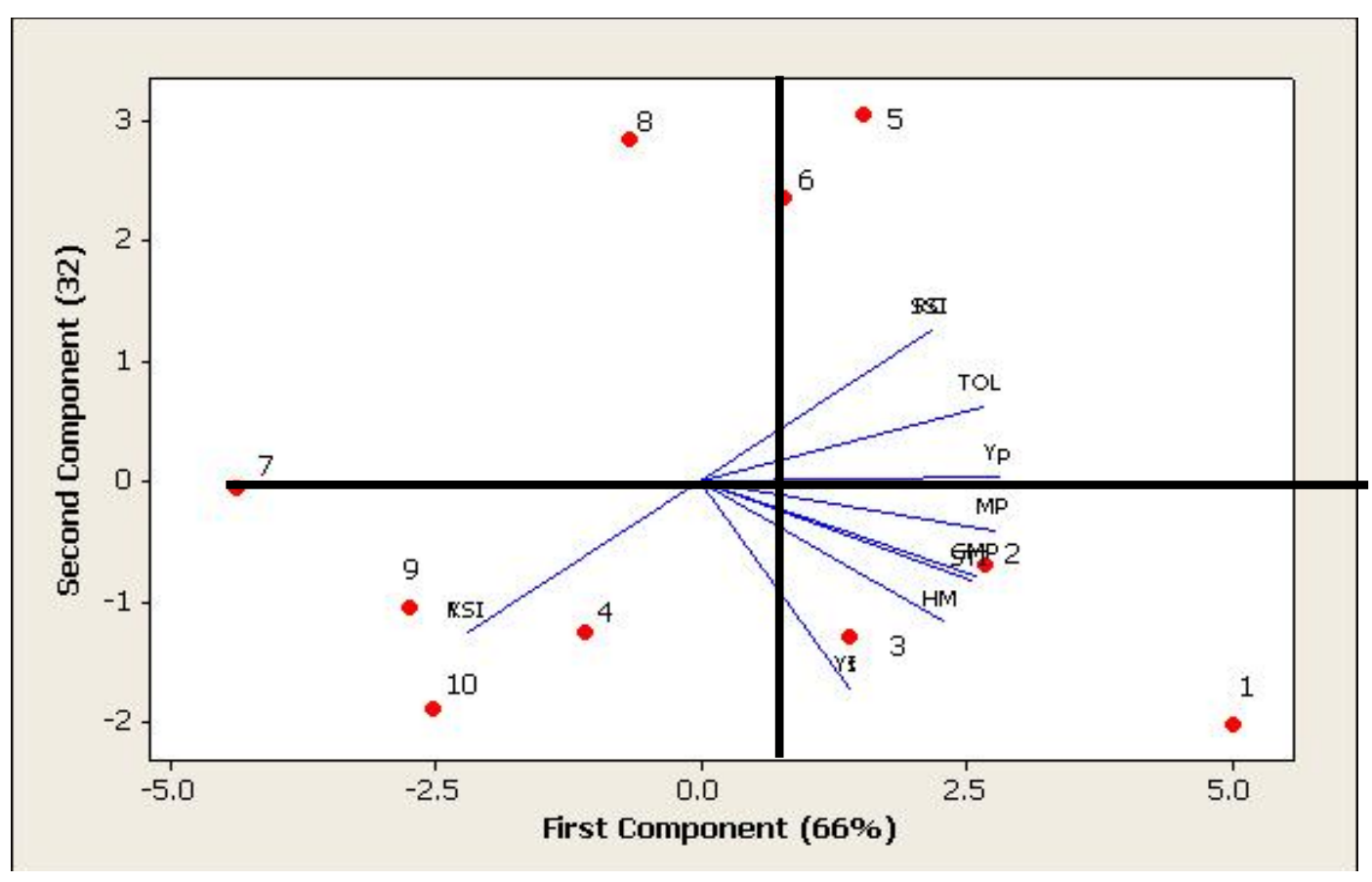

شكل ا - ترسيه باى يلات بر اساس دو مؤلفه اول با استفاده از شاخصهاى تحمل به تنش خشكى

Figure1. Bi plot drawing based on the first two components using drought tolerance indices

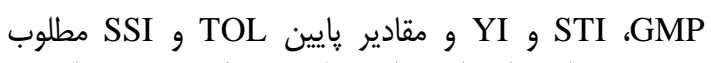

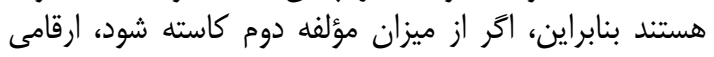
انتخاب مىشوند كه داراى MP و

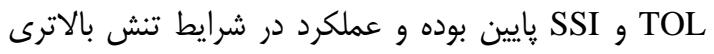

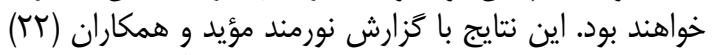

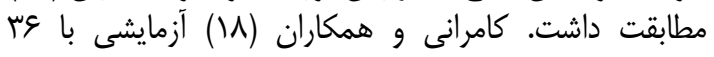

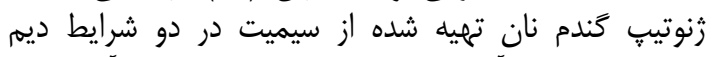

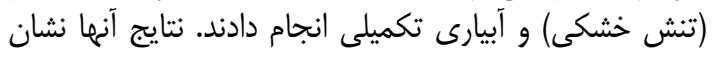

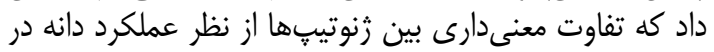

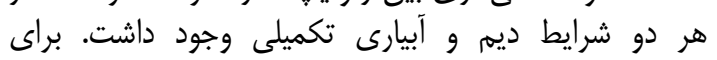

مؤلفه اول بهدليل داشتن ضرايب بالا و مثبت براى تمام

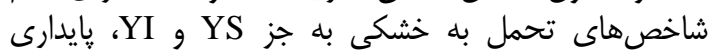

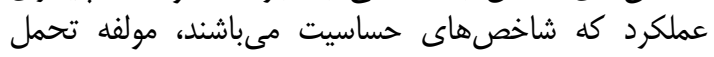

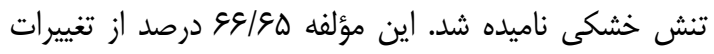

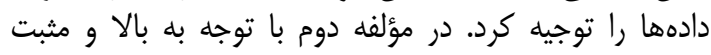

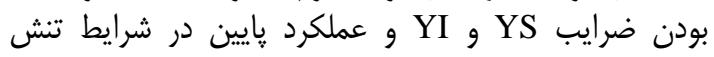

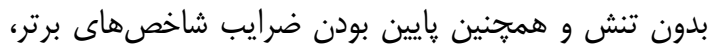

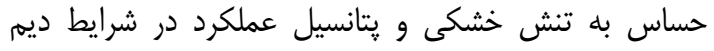

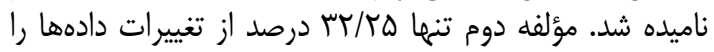

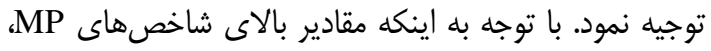


تجزيه خوشهاى بر اساس شاخصهاى تحمل به

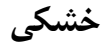

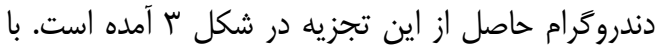

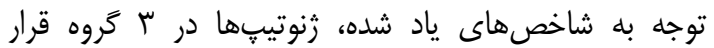

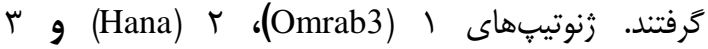
(Aria(stork))

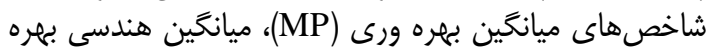

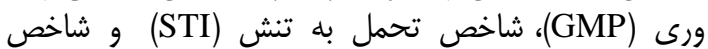

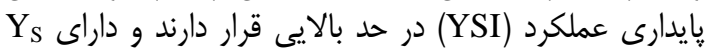

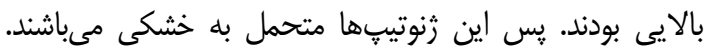

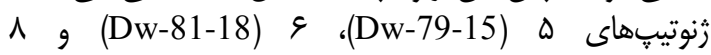
YP نيز در يك كروه قرار كرفته كه داراي (18/A/maragheh)

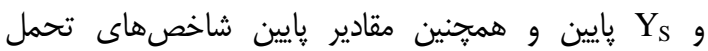

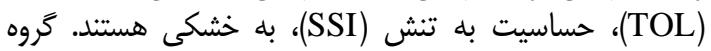

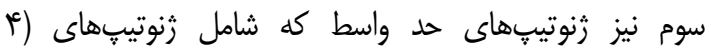
و(Dw-73-13) (15/A/Maragheh)V، (Saji(cheek))

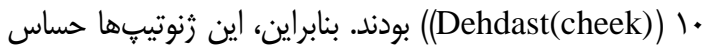

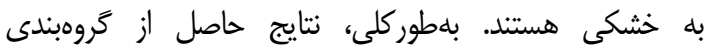

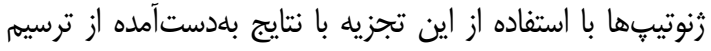
باى يلات كَابريل مطابقت نشان دان إدان

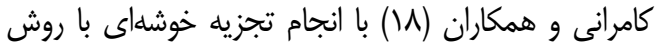

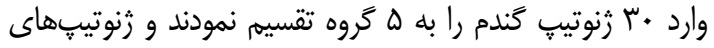

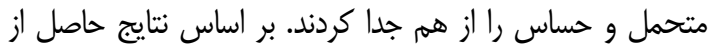

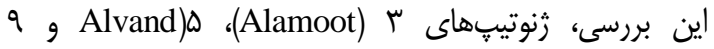
(F-Gy54)

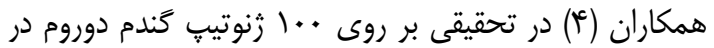

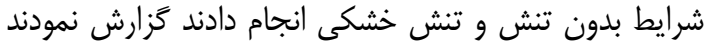

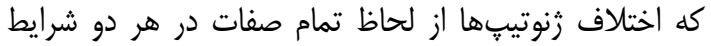

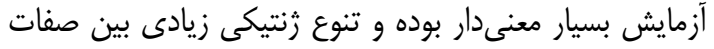

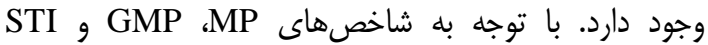
ثنوتيتهاى (F) (Y)

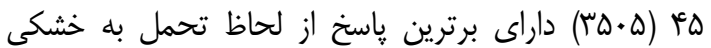

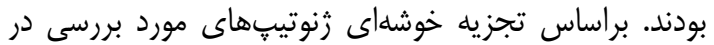

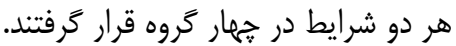

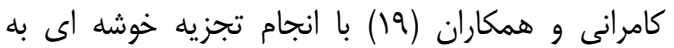

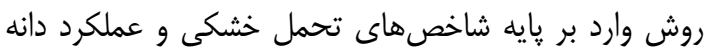

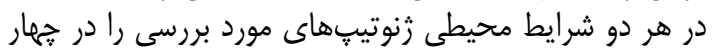

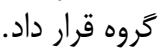

شناسائى زنوتيبهاى متحمل به خشكى، از شاخصهاى

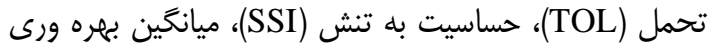

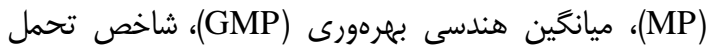

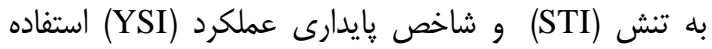

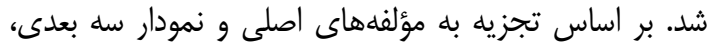

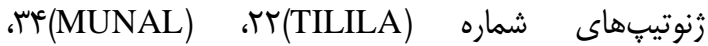

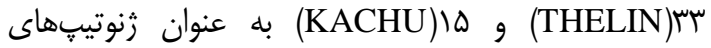

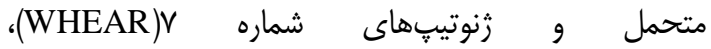
(PBW) و I I I I I (LAKHISH) به ته بنش خشكى شناخته شدند.

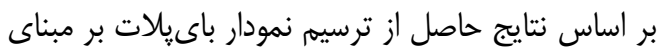

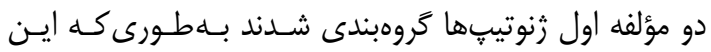

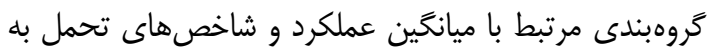

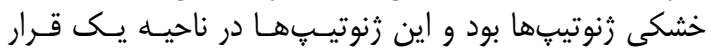

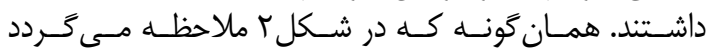

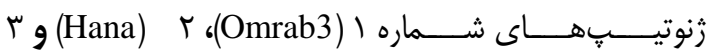
(Aria(stork))

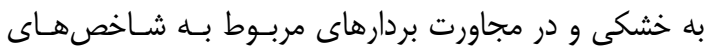

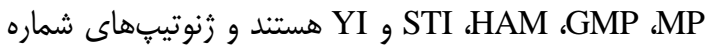
(18/A/maragheh)^ و (Dw-81-18)\&، (Dw-79-15) ه

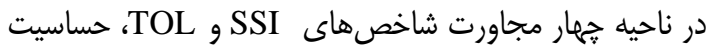

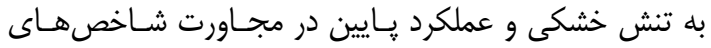

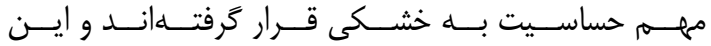

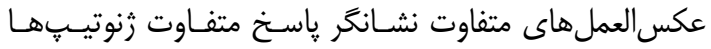

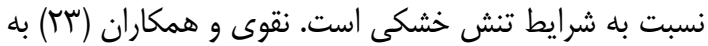

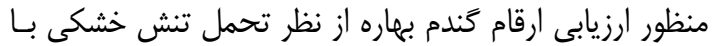

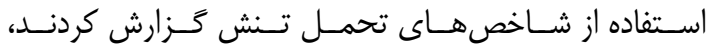

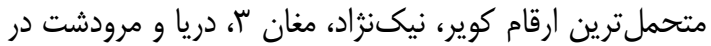

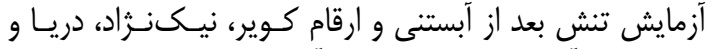

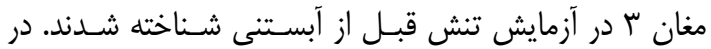

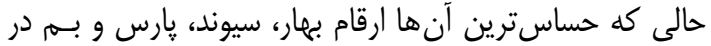

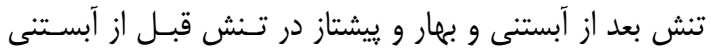

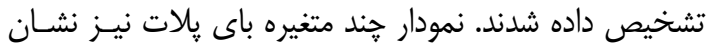

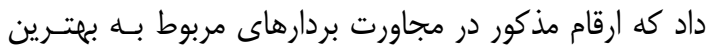

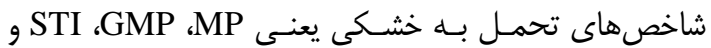

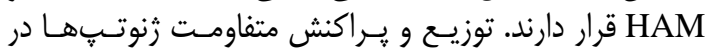

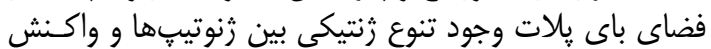

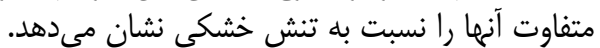




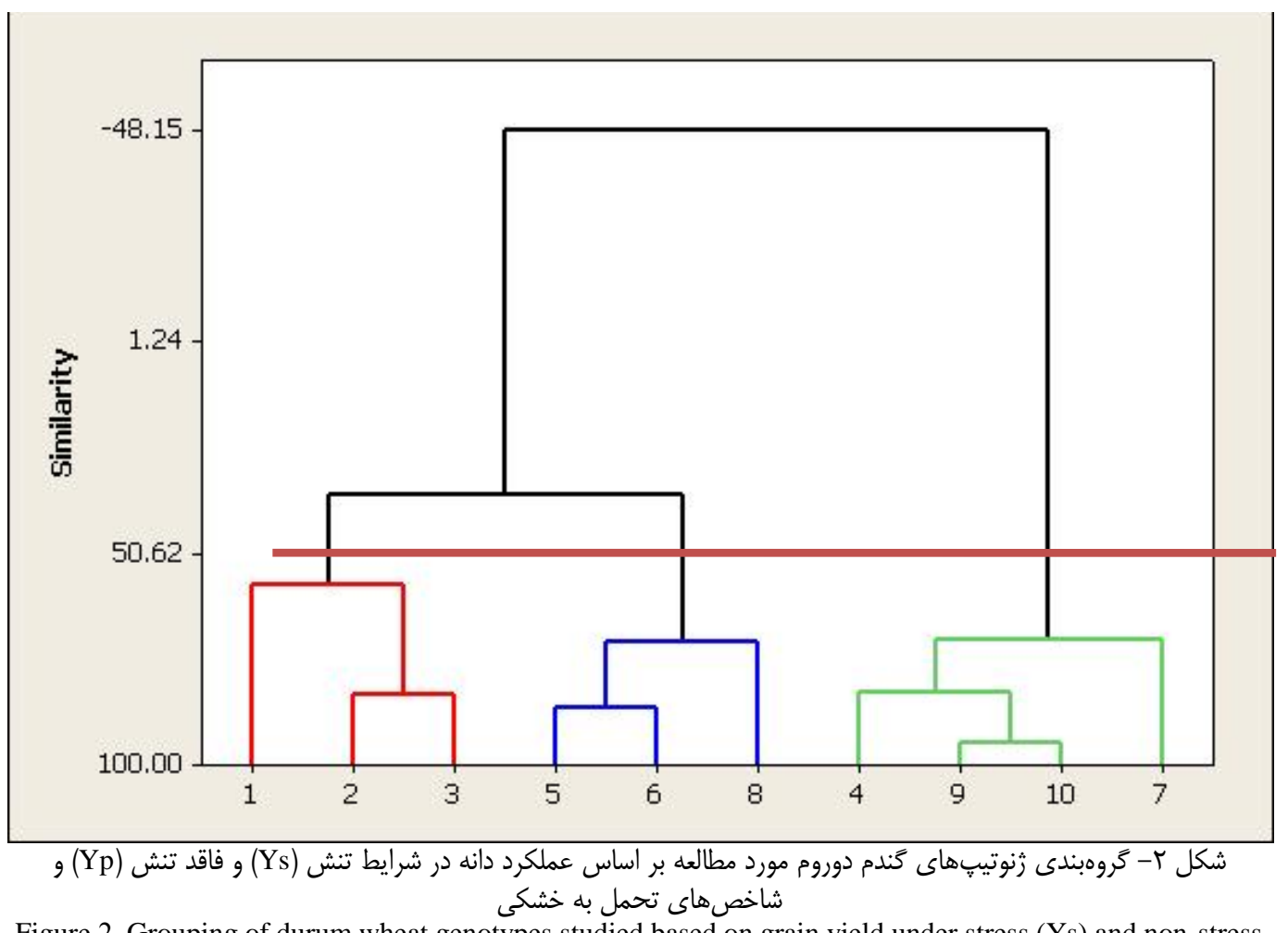

Figure 2. Grouping of durum wheat genotypes studied based on grain yield under stress (Ys) and non-stress conditions (Yp) and drought resistance indices

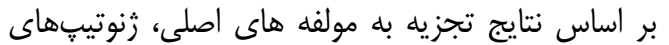

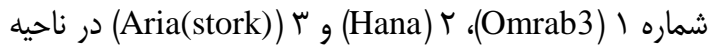

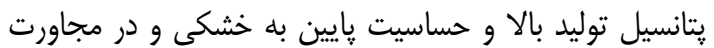

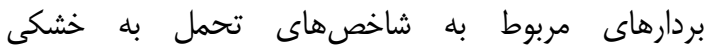

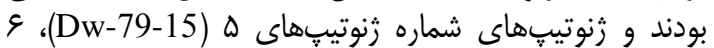

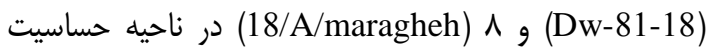
به تنش خشكى و عملكرد يايين در مجاورت شاخصهاي

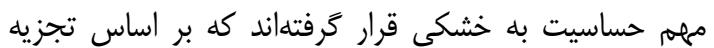

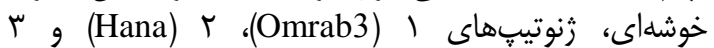

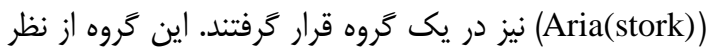

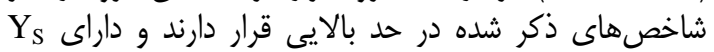

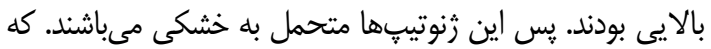

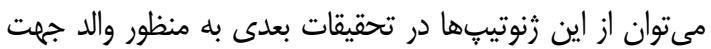
برناملهاى اصلاحى استفاده نمود.
بلهور كلى نتايج حاصل از اين يزوهش نشان داد كه إنه بين

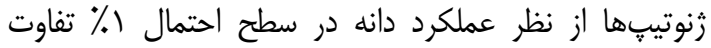

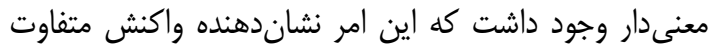

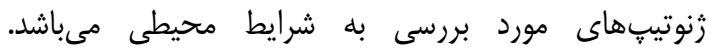

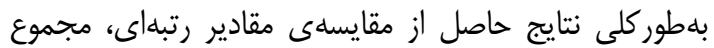

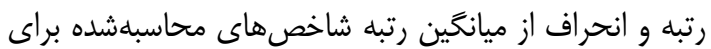

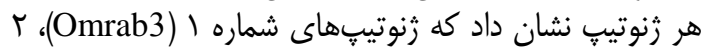

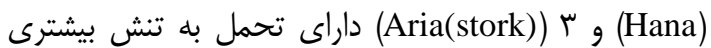
نسبت به ساير زنوتي»هاى مورد مطالعه بودند.

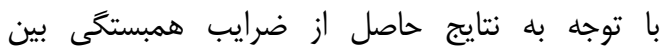

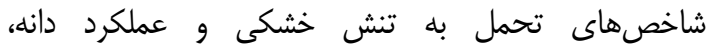

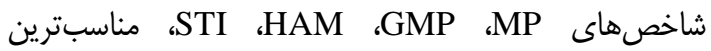

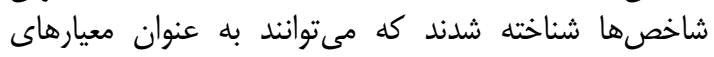

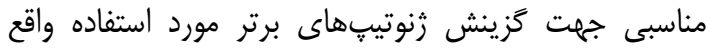
شوند.

منابع

1. Aghaee-Sarbarzeh M., R. Rajabi, R. Haghparast and R. Mohammadi. 2009. Evaluation of Proline Content, Cell Membrane Damage, and Drought Tolerance in Durum Wheat (Triticum turgidum var. durum) Genotypes under Controlled Conditions. Seed and Plant Production Journal, 25(3): 345-352. doi: 10.22092/sppj.2017.110382

2. Ali, M.B. and A.N. El-Sadek. 2016. Evaluation of drought tolerance indices for wheat (Triticum aestivum L.) under irrigated and rainfed conditions. Communications in Biometry and Crop Science, 11: 77-89.

3. Amini, M. 2010. Overview of Macaroni Production in the World and Iran. Semolina, 1: 48-1.

4. Bakhshaeshi Gheshlagh, M. and M. Shekarchi. 2016. Evaluation of bread wheat genotypes using drought tolerance indices. Journal of Crop Breeding, 7(16) :49-59URL: http://jcb.sanru.ac.ir/article-1500-fa.html (In Persian). 
5. Bihamta, M., M. Shirkavand, J. Hasanpour and A. Afzalifar. 2018. Evaluation of Durum Wheat Genotypes under Normal Irrigation and Drought Stress Conditions. Journal of Crop Breeding, 9(24): 119-136.URL: http://jcb.sanru.ac.ir/article-1-944-fa.html (In Persian).

6. Bouslama, M. and Schapaugh, W.T. 1984. Stress tolerance in soybean. I: Evaluation of three screening techniques for heat and drought tolerance. Crop Science, 24: 933-937.

7. Farshadfar, E. and P. Elyasi. 2012. Screening quantitative indicators of drought tolerance in bread wheat (Triticum aestivum L.) landraces. European Journal of Experimental Biology, 2(3): 577-584.

8. Farshadfar, E., M.M. Pour Siahbidi and Pour A.R. Aboughadareh. 2012. Repeatability of drought tolerance indices in bread wheat genotypes. International Journal of Agriculture and Crop Science, 4(13): 891-903.

9. Fayaz, N. and A. Arzani. 2011. Moisture stress tolerance in reproductive growth stage in triticale (X Triticosecale Wittmack) cultivars under field conditions. Journal of Crop Breeding, 1(1): 1-12.

10. Fernandez, G.C.J. 1992. Effective selection criteria for assessing stress tolerance. In: Kuo. C.G. (Ed). Proceedings of the international symposium on adaptation of vegetables and other food crops in temperature and water stress, publication, Taina, Taiwan. 13-16 Aug. Chapter, 25: 257-270.

11. Fischer, R. and R. Maurer. 1987. Drought resistant in spring wheat cultivars. I: Grain yield response. Australian Journal of Agricultural Research, 29(5): 895-97.

12. for yield in stress and non-stress environment. Crop Science, 21(6): 43-46.

13. Gabriel, K.R. 1971. The biplot graphical display of matrices with applications to principal component analysis. Biometrika, 58(3): 453-467.

14. Gavuzzi, P., F. Rizza, M. Palumbo, R.G. Campaline, G.L. Ricciaroli and B. Borghi. 1997. Evalution of field and laboratory predictors of drought and heat tolerance in winter cereals. Canadian Journal of Plant Science, 77(4): 523-531.

15. Golabadi, M. and A. Arzani. 2002. Evaluation of Grain Quality Traits, Glutenin Subunits and Their Relationship in Durum Wheat. JWSS, 6(3):189-203URL: http://jstnar.iut.ac.ir/article-1-224-fa.html

16. Golabadi, M. and A. Arzani. 2003. Study of Genetic Variation and Factor Analysis of Agronomic Traits in Durum Wheat. JWSS, 7(1) :115-127URL: http://jstnar.iut.ac.ir/article-1-388-fa.html

17. Haji Mohammad Ali Jahromi, M., M. Khodarahmi, A.R. Mohammadi, A. Mohammadi and R. Sadegh Gol Moghadam. 2010. Phenotypic flexibility analysis of promising durum wheat genotypes in dry and hot climates in southern Iran. Journal of Agronomy, 6(3): 61-70 (In Persian).

18. Kamrani, M., A. Farzi Shiri and M. Evaluation. 2017. of Drought Tolerance in Some Wheat Genotypes using Drought Tolerance Indices. Journal of Crop Breeding, 9(23) :9-17URL: http://jcb.sanru.ac.ir/article-1-869-fa.html

19. Kamrani, M., A. Mehraban, M. Shiri. 2018. Identification of Drought Tolerant Genotypes in Dryland Wheat using Drought Tolerance Indices. Journal of Crop Breeding, 10(28): 13-26 URL: http://jcb.sanru.ac.ir/article-1-891-fa.html

20. Kristin, A.S., R.R .Senra, F.I.. Perez, B.C. Enriquez, J.A.A. Gallegos, P. R. Vallego, N. Wassimi and J.D. Kelley. 1997. Improving common bean performance under drought stress. Crop Science, 37: 43-50.

21. Moosavi, S.S., B. Yazdi Samadi, M.R. Naghavi, A.A. Zali, H. Dashti and A. Pourshahbazi. 2008. Introduction of new indices to identify relative drought tolerance and resistance in wheat genotypes. Desert, 12: 165-178.

22. Naghavi, M.R., M. Moghaddam, M. Torchi and M.R. Shakiba. 2016. Evaluation of spring wheat cultivars based on drought resistance indices. Journal of Crop Breeding. . Journal of Crop Breeding, 8(17): 207-192URL: http://jcb.sanru.ac.ir/article-1-611-fa.html

23. Naghdipoor, A., M. Khodarahmi, A. Porshahbazi and M. Ismailzade. 2011. Factor analysis for grain yield and other traits of durum wheat. Journal of Agronomy, 7(1): 89-96 (In Persian).

24. Nourmand Maid, F., M.A. Rostami, M.R. Confectioners. 2001. Investigation of Morphophysiological Traits of Bread Wheat and Their Relationship with Yield in Stress and Non-Drought Conditions. Iranian Agricultural Science, 32(4): 794-785.

25. Omid Bakhsh FARD, M., M. Naghavi, M. Mardi, M. Bihamta, M. Kazemi and S. Pirseyedi. 2009. A Study of Genetic Diversity in Durum Wheat (Triticum turgidum) using Microsatellite Markers. Iranian Journal of Field Crop Science, 40(2).

26. Ramirez-Vallejo, P. and J.D. Kelly. 1998. Traits related to drought resistance in common bean. Euphytica, 99(2): 127-136.

27. Rao, V.S. 2008. Mapping and validation of a major QTL for yellow pigment content on 7AL in durum wheat

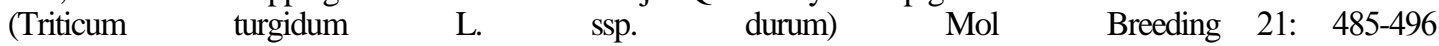

28. Rosielle, A.A. and J. Hamblin. 1981. Theoretical Aspects of Selection for Yield in Stress and Nonstress Environments. Crop Sciences, 21: 943-946.

29. Sadeghzadeh Ahari, D. 2007. Evaluation of Drought Tolerance of Promising Durum Wheat Genotypes. Journal of Crop Sciences, 8(1): 45-30.URL:http://agrobreedjournal.ir/article-1-302fa.html

30. Sio-se Mardeh, A., A. Ahmadi, K. Poustini and V. Mohammadi. 2006. Evaluation of drought resistance indices under various environmental conditions. Field Crops Research, 98(2-3): 222-229. 


\title{
Evaluation of Drought Tolerance in Durum Wheat Genotypes using Drought Tolerance Indices
}

\author{
Hoshang Rahmati ${ }^{1}$, Ali Nakhzari Moghadam ${ }^{2}$, Ali Rahemi Karizaki ${ }^{3}$ and \\ Zeynab Evarsaji ${ }^{4}$

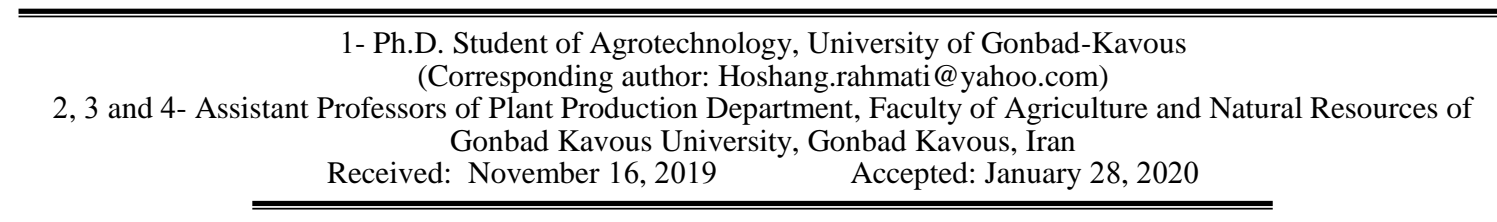

\begin{abstract}
Drought is one of the most important factors limiting the growth and production of crops in most parts of the world and Iran and affects as a multidimensional stress on plants at different levels. In order to study drought tolerance of 10 durum wheat genotypes, an experiment was conducted in a randomized complete block design with three replications in stress field in Kouhdasht city. Results of analysis of variance showed that the response of the studied genotypes was different for grain yield and the possibility of selection of genotypes based on each of the traits. Comparison of numerical values and rank of each genotype according to drought tolerance indices showed that based on drought tolerance indices according to seed yield of genotypes 1 (Omrab3), 2 (Hana) and 3 (Aria (stork) as drought tolerant and genotypes, Other genotypes were identified as susceptible genotypes. The results of correlation coefficients between indices showed that MP, GMP, HAM, STI indices had positive and significant correlation at $1 \%$ probability level with grain yield in stress and non-stress conditions. There were some, so it can be said that these indices are the best indices for identifying superior genotypes. Principal component analysis results showed that the first two components accounted for about $99 \%$ of the data variation. According to Gabriel's biplot chart, the studied genotypes were classified into two high yield potential and low yield potential areas, with genotypes 1 (Omrab3), 2 (Hana) and 3 (Aria (stork) being adjacent to vectors. Drought tolerance was identified as superior genotypes. Cluster analysis based on superior indices separated the studied genotypes into three general groups, which were in good agreement with the results of Gabriel biplot plotting.
\end{abstract}

Keywords: Wheat, Bipot, Stress 